University of Nebraska - Lincoln

DigitalCommons@University of Nebraska - Lincoln

U.S. National Park Service Publications and

Papers

National Park Service

$11-26-2019$

\title{
Material and Seismic Assessment of the Great House at Casa Grande Ruins National Monument, Arizona
}

Douglas W. Porter

Anjali Mehrotra

Matthew J. DeJong

Angelyn Bass

Matthew Guebard

See next page for additional authors

Follow this and additional works at: https://digitalcommons.unl.edu/natlpark

Part of the Environmental Education Commons, Environmental Policy Commons, Environmental

Studies Commons, Fire Science and Firefighting Commons, Leisure Studies Commons, Natural Resource

Economics Commons, Natural Resources Management and Policy Commons, Nature and Society

Relations Commons, Other Environmental Sciences Commons, Physical and Environmental Geography

Commons, Public Administration Commons, and the Recreation, Parks and Tourism Administration

Commons

This Article is brought to you for free and open access by the National Park Service at DigitalCommons@University of Nebraska - Lincoln. It has been accepted for inclusion in U.S. National Park Service Publications and Papers by an authorized administrator of DigitalCommons@University of Nebraska - Lincoln. 
Authors

Douglas W. Porter, Anjali Mehrotra, Matthew J. DeJong, Angelyn Bass, Matthew Guebard, and John Ochsendorf 


\title{
Material and Seismic Assessment of the Great House at Casa Grande Ruins National Monument, Arizona
}

\author{
Douglas W. Porter ${ }^{1}$; Anjali Mehrotra ${ }^{2}$; Matthew J. DeJong, A.M.ASCE${ }^{3}$; \\ Angelyn Bass ${ }^{4}$; Matthew Guebard ${ }^{5}$; and John Ochsendorf ${ }^{6}$
}

\begin{abstract}
The authors characterized earthen wall materials and plasters in a mid-fourteenth-century Hohokam great house at Casa Grande Ruins National Monument (Arizona) and assessed the seismic susceptibility of its puddled earth walls. Characterization included determining the microstructure, microcomposition, porosity, aggregate mineralogy, and identification of phases in the binding matrix for each of 36 samples and reconstructing plaster technologies, including material selection, preparation, and application sequences. Findings support the ideas that earthen materials were manipulated to optimize their performance to suit the unique site conditions and needs of the ancient people using the structure and included finishes that were unusual in southwestern sites from this time period. By using a new set of tools that integrate the complicated geometry of individual wall segments as captured in light detection and ranging (LiDAR) scans (models were generated in Rhino version 5) with the dynamic analysis of rocking mechanisms (tools for this analysis were developed in Rhino), seismic collapse assessment was used to identify the most vulnerable parts of the building to earthquake loading and provided an initial evaluation of the seismic overturning capacity of these wall segments. DOI: 10.1061/(ASCE)AE.1943-5568.0000371. (C) 2019 American Society of Civil Engineers.
\end{abstract}

\section{Introduction}

The great house at Casa Grande Ruins National Monument, occupied circa 1350-1450 CE (Wilcox and Sternberg 1981), is a fourstory Hohokam structure of puddled earth. The great house was built by ancestral Native Americans known to archaeologists as the Hohokam culture. The Hohokam consisted of ancestral Native American groups living in the Gila and Salt River drainages in Arizona between 200 or $500 \mathrm{CE}$ and $1450 \mathrm{CE}$. The great house was built during the Civano phase (1300-1450 CE) and is associated with what archaeologists call the Classic Period defined by the appearance of walled compounds, public architecture, population aggregation, increasing use of inhumation burials, and the appearance of polished red and polychrome ceramics (Wilcox and Sternberg 1981; Abbott et al. 2003; Guebard et al. 2018). At Casa Grande (a unit in the National Park system), the great house is the largest of the structures located within a series of walled compounds that include an elliptical ball court, a platform mound, and other earthen structures (Fig. 1) (Van Valkenburgh 1962; Guebard et al. 2018). The structure consists of 11 earthen plastered rooms and at least four open areas on the rooftops. The walls are

\footnotetext{
${ }^{1}$ Assistant Research Professor, Dept. of Civil and Environmental Engineering, Univ. of Vermont, Burlington, VT 05405 (corresponding author). Email: douglas.porter@uvm.edu

${ }^{2}$ Postdoctoral Researcher, Dept. of Civil and Environmental Engineering, Univ. of California-Berkeley, Berkeley, CA 94720.

${ }^{3}$ Assistant Professor, Dept. of Civil and Environmental Engineering, Univ. of California-Berkeley, 777 Davis Hall, Berkeley, CA 94720.

${ }^{4}$ Research Assistant Professor, Dept. of Anthropology, Univ. of New Mexico, Santa Fe, NM 87505. ORCID: https://orcid.org/0000 $-0002-9377-5897$

${ }^{5}$ National Park Service, 527 South Main St., Camp Verde, AZ 86322.

${ }^{6}$ Professor, Dept. of Civil and Environmental Engineering/Architecture, Massachusetts Institute of Technology, Cambridge, MA 02139.

Note. This manuscript was submitted on March 31, 2018; approved on March 6, 2019; published online on November 26, 2019. Discussion period open until April 26, 2020; separate discussions must be submitted for individual papers. This paper is part of the Journal of Architectural Engineering, (C) ASCE, ISSN 1076-0431.
}

more than $1.25 \mathrm{~m}$ thick and are remarkably plumb and smooth, and the interior walls are covered with earthen plasters with red washes. Architectural features include viga sockets and other elements related to floor and roof construction, wall openings with plastered jambs and sockets for wood lintels, and observation holes and related features that may originally have been intended for making astronomical observations. This earthen complex was the first archaeological site to be stabilized by the Smithsonian Institution in 1891 and the first in the United States to be protected by Congress in 1892 (Mindeleff 1896).

Ceramics found at Casa Grande suggest the site was occupied until the mid-fifteenth century (Steen 1965; Wilcox and Shenk 1977). By the late-seventeenth century, the roof and floor structures had collapsed, leaving the tall earthen walls relatively unbraced. Since the publication of Cosmos Mindeleff's first report on the Casa Grande in 1896, site stewards have been concerned with its structural stability. Issues include substantial losses at the bases of walls; columnification of the walls due to large vertical throughcracks; discretization of unsupported masonry above wall openings, where wood lintels have been lost (potentially leading to the collapse of these blocks); and seismic vulnerability of the structure. The collapse of a large wall segment on the east perimeter wall in 1887 is thought to have been the result of an earthquake with an estimated magnitude of 7.2 (Wilcox and Sternberg 1981). Mindeleff (1896) installed wood beams and iron tie rods in the 1890 s to stabilize walls, and more recent intervention proposals have included reinforcement by using an extensive system of wall cores and reinstatement of wood structural elements.

Since 2012, the park and the Department of Anthropology at the University of New Mexico have been engaged in collaborative research focused on the completion of an architectural analysis of the great house, a reassessment of the building's physical condition, the characterization of the ancient wall materials and plasters, nondestructive testing to characterize the fracture network in the earthen walls, and a structural assessment of seismic vulnerability. This paper describes the analytical methods and results of this assessment. By gaining a better understanding of period building technology, physical changes to the building materials over time, 


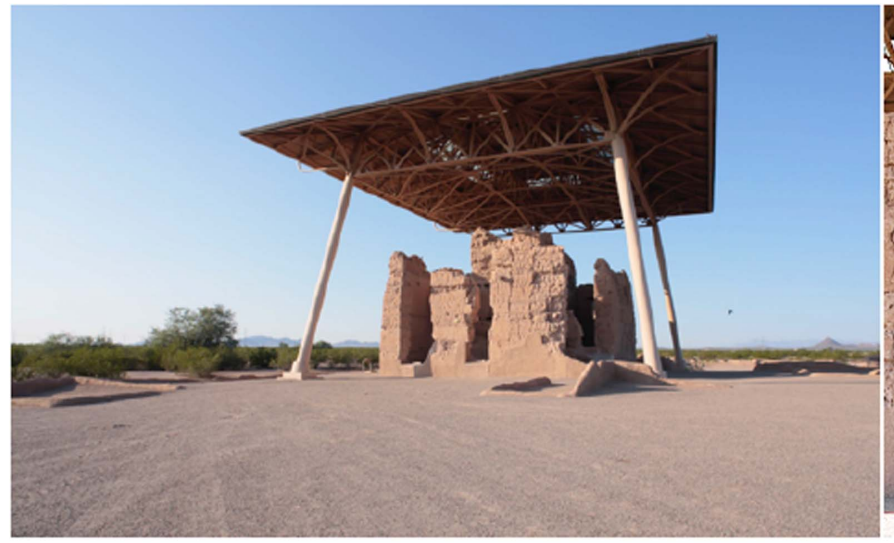

(a)

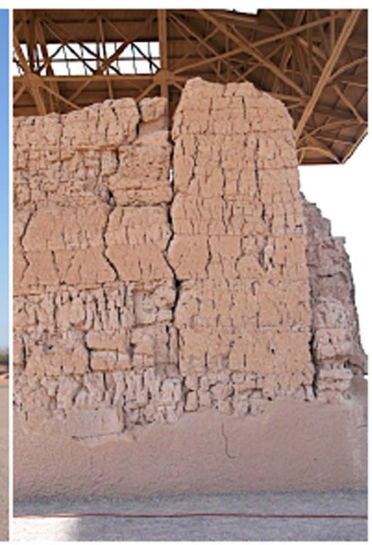

(b)

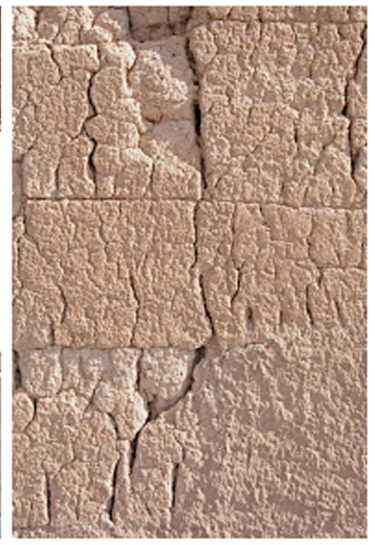

(c)

Fig. 1. Great House at Casa Grande National Monument: (a) four-story Hohokam structure of puddled caliche occupied from ca. 1350-1450 CE. Note cambered surfaces of upper walls; and (b) and (c) east perimeter wall showing coursing, desiccation fractures, and large vertical cracks that divide wall into several segments. Larger through-wall cracks are possibly results of earthquakes. (Images courtesy of Neil Dixon.)

and the vulnerability of individual wall segments, future interventions can be minimized and directed specifically where they are needed, protecting the material integrity and physical authenticity of the structure.

\section{Wall Construction}

The earthen materials used in the construction of the great house walls include a core, or hearting, of puddled caliche covered with leveling coats of earthen plaster of varying thickness to establish the wall planes. Interior walls are covered with fine finish plasters and washes (Bass et al. "Condition Assessment and Treatment Planning for the Great House, Casa Grande Ruins National Monument" 2014, unpublished report; Bass et al. 2017). There are no finishes on the great house exterior; instead, coursing and construction episode boundaries are visible from the outside, along with desiccation fractures that appeared following construction. The characteristics of hearting, leveling coats, and finish plasters and washes are listed in Table 1. Techniques for characterizing earthen materials generally require large samples. By combining optical microscopy, X-ray diffraction analysis, scanning electron microscopy, and energy dispersive X-ray spectroscopy, investigators were able to identify clays and aggregates, secondary minerals, and soluble salts. Analyzing back-scattered electron-scanning electron microscope (BSE-SEM) micrographs with software from JEOL 5700 SEM software (Oxford Instruments PLC, Oxfordshire, UK) and Image J version 1.51 yielded information about porosity, volumetric proportions of binder and aggregate materials, and particle size and morphology. In this way, investigators were able to produce detailed characterizations of the earthen materials at Casa Grande by using microsamples; for additional information on methods, see Bass et al. (2017).

In selecting earthen materials, the builders intentionally targeted subsurface calcium carbonate accumulations (Wilcox and Shenk 1977; Bass et al. 2017), and samples of hearting and leveling plaster contain abundant authigenic carbonate nodules along with silicate clays. In constructing the walls of the Casa Grande, the caliche was apparently prepared by wetting and kneading it into a plastic mass, and individual lenses of the wet material were deposited on the walls and smoothed by hand. These lenses were built up to form individual courses, denoted by well-formed horizontal and subhorizontal seams at course boundaries (Wilcox and Shenk 1977).
Courses vary in height but are typically $61-76 \mathrm{~cm}$ (24-30 in.) tall. As the material dried, it shrank, producing a well-ordered system of cracks. In addition to this system of cracks forming on the desiccation of the wall material, there are vertical cold joints that can be recognized by offsets in horizontal seams on either side (Wilcox and Shenk 1977) of a vertical joint. The weathered exterior wall surfaces are characterized by a rind, $0.3-0.6 \mathrm{~mm}$ thick, composed principally of smectite and illite clays with traces of iron that contribute the characteristic red color. The void spaces in the near surface retain the scalenohedral shape of calcite crystals leached from this zone (Fig. 2).

Interior walls were finished with one or more coats of fine finish plaster, followed by clay- and gypsum-based washes (Fig. 3). Finegrained finish plasters consist of semirounded fine-to-medium sands (largest aggregates $\leq 1.5 \mathrm{~mm}$ ) in a clay and calcite matrix. All interior wall surfaces were finished similarly with clay-based (illite and palygorskite) and gypsum washes that contributed the red color and characteristic sheen. The red washes also contained a distinctive calcium phosphate component that would appear to be the result of the admixture of ash or bone to the wash. Interior plasters are largely intact, and the consistency of the finishes, the use of the materials, and the singular aesthetic effect vary considerably from the earthen finishes observed at most Southwestern sites from the same time period.

In hearting, leveling coats, and finish plasters, secondary calcite is abundant. Secondary calcite appears as scalenohedral crystals $(1-5 \mu \mathrm{m})$ precipitated in the pore space, and agglomerations of calcite crystals forming grains that are up to $50 \mu \mathrm{m}$ in diameter (Fig. 4). Total optical porosity in these calcite-rich zones is frequently between $6.5 \%$ and $20 \%$, though in several of the samples examined by investigators, optical porosity is less than $5 \%$, with calcite accounting for more than half of the material (Bass et al., unpublished report, 2014; 2017). One would expect that imbibition rates would be low and that the calcite bonds would yield a relatively strong material; earlier characterizations reported values greater than 400 psi for the compressive strength of the wall materials (Vick 1973).

However, in addition to the desiccation fractures that cover the building exterior, there are long vertical cracks, frequently located along intersecting walls, that have resulted in the division of the walls into a series of tall, relatively slender columns. Cracks also appear at the tops and ends of walls that are oriented parallel to wall 
surfaces (Fig. 5). One would expect that cracks that formed on the desiccation of the clay would be surficial, but it is not as clear whether axial cracks appearing at the tops and ends of walls are of the same sort or whether they occur along the boundaries of hearting and leveling coats and form an extensive interconnecting network interior to wall surfaces. In addition, there are substantial losses that appear at wall bases and along rows of viga sockets that carried floor and roof frame members that effectively reduce wall width by up to $50 \%$ in places.

\section{Seismic Vulnerability Analysis}

Previous research into the tools and procedures for the seismic vulnerability analysis of historical construction has varied from the formulation of indicator-based methods to the development of more mechanics-based approaches. Regarding the former, an indicatorbased method for the seismic vulnerability assessment of archaeological sites was recently developed by Minos-Minopoulos et al. (2017), which combines spatial parameters, such as the susceptibility of the sites to secondary earthquake; environmental effects, such as soil liquefaction and landslides and tsunamis; with temporal parameters, such as physical vulnerability, economic importance, and visitor density; to determine the overall vulnerability level. Conversely, most mechanics-based approaches tend to focus on the structures themselves-modeling local elements susceptible to collapse as rigid blocks - and comprise a mixture of rocking and kinematic analysis methods - a complete review of which can be found in Casapulla et al. (2017).

However, a number of these approaches depend on a priori definition of the collapse mechanisms, which, in the case of more complex structural geometries as found in archaeological sites, is often hard to predict. To this end, a new numerical procedure was developed by Galassi et al. (2018a, b) and implemented within the software BrickWORK (Galassi and Paradiso 2014), which models archaeological ruins as an assemblage of rigid macroblocks connected through a series of axial and shear links and conducts an incremental nonlinear analysis to determine the corresponding (local) collapse mechanism. Moreover, the load factor required to activate this mechanism is also used to calculate the corresponding spectral acceleration, which, in turn, is used to quantify the seismic vulnerability of the structure. However, for rocking structures in particular, this force-based approach, while useful for identifying collapse mechanisms, can lead to overconservative results when predicting their actual out-of-plane capacity (Casapulla et al. 2017). In fact, dynamically loaded masonry structures have been found to sustain accelerations over and above the limit determined by using quasi-static approaches. Consequently, displacement-based approaches, which quantify seismic vulnerability, in terms of maximum displacements/rotations, have been found to be more realistic tools for the assessment of the dynamic out-of-plane response of these structures (Casapulla et al. 2017) — which is also the approach employed in this paper.

The seismic vulnerability assessment of archaeological ruins is a complex problem-due, in part, to the large number of inherent unknowns - and numerous different numerical and analytical tools and procedures have been used to analyze a variety of case studies in the literature. A limited number of examples are cited here to exemplify different approaches. Ruggieri et al. (2018) conducted both pushover and kinematic analyses on a portion of the Stabian Baths in Pompeii, which were used to propose a timeline reconstruction of the seismic events that occurred in the first century AD. Giamundo et al. (2014) used a combination of numerical (discrete element) modeling and an Italian code-based method to 


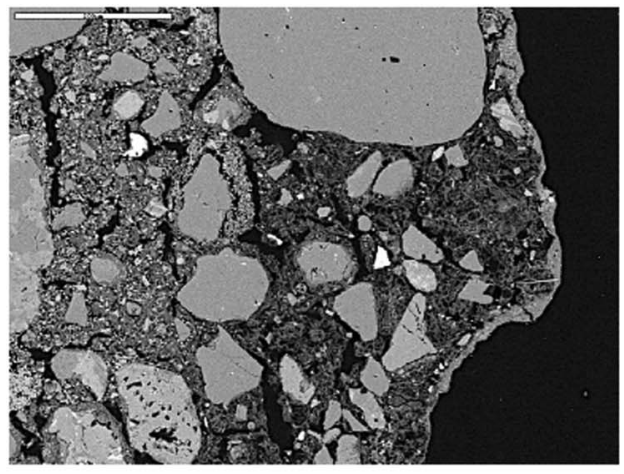

(a)

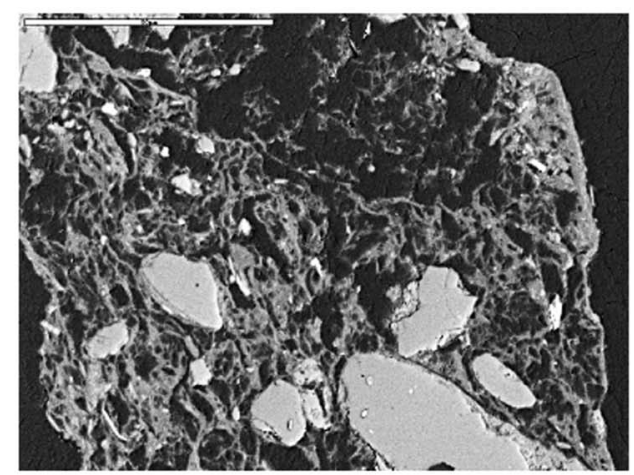

(b)

Fig. 2. (a) BSE-SEM of exterior wall material showing a thin layer of fine-grained material at surface (scale bar $=200 \mu \mathrm{m}$ ); and (b) clay-rich layer with distinctive texture from leaching of calcite. Pore structure in this zone retains scalenohedral shape of leached calcite (scale bar $=50 \mu \mathrm{m}$ ).

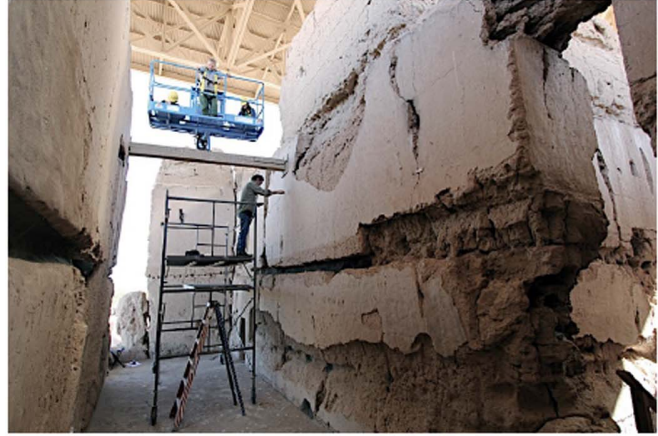

(a)

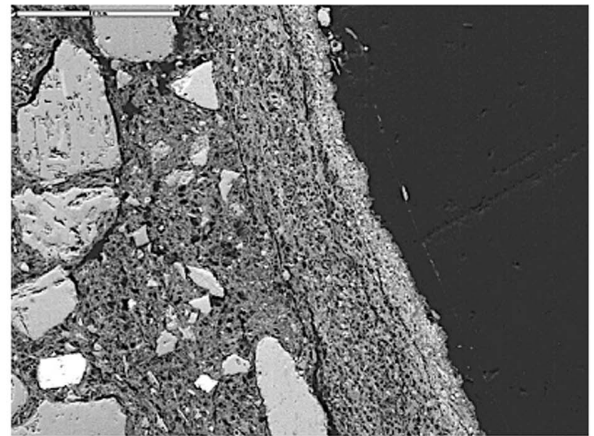

(b)

Fig. 3. Interior of Casa Grande: (a) Stack A (image by authors) finished with plaster and red washes. Long horizontal rows of viga sockets are related to floor and roof construction materials that have been lost; and (b) BSE-SEM of finish plaster substrate (image left), clay wash (image center, $50 \mu \mathrm{m}$ thick), and gypsum wash (image right, $\leq 20 \mu \mathrm{m}$ thick) (scale bar $=100 \mu \mathrm{m}$ ).

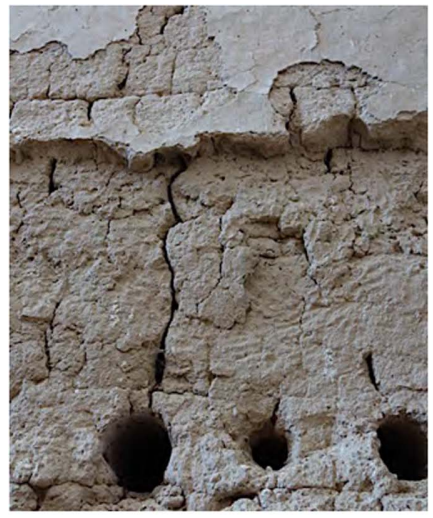

(a)

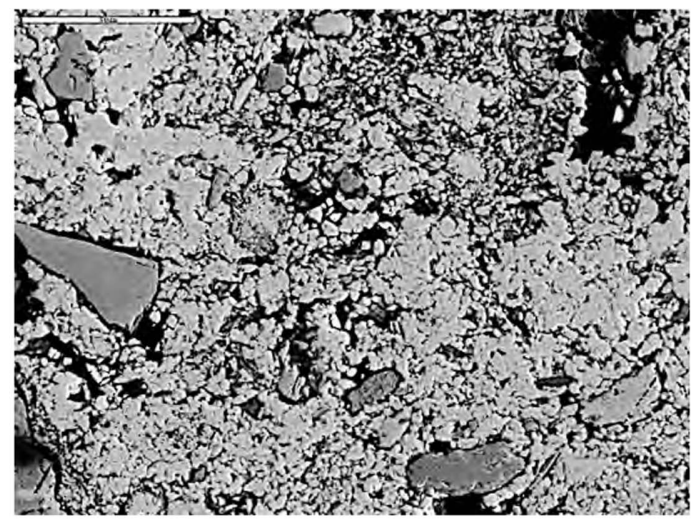

(b)

Fig. 4. Interior elevation of west wall of Room B: (a) handprint impressions on hearting material (lower half). Leveling plaster is $2-3 \mathrm{~cm}$ thick, and finish plaster and washes are visible at top of the image (image by authors); and (b) BSE-SEM of the hearting. Heavy accumulations of secondary calcite; at this scale, fractures account for most of open porosity (scale bar $=50 \mu \mathrm{m})$.

analyze the seismic vulnerability of a two-story colonnade in Pompeii to preserve the safety of the structure as well as study the effects of the degradation of the archaeological material. Similarly, a combination of detailed finite-element modeling with a more simplified structural analyses by Andreini et al. (2013) enabled conclusions to be drawn regarding the seismic safety and stability of the Mediceo Aqueduct in Pisa, Italy. A more comprehensive methodology for the seismic assessment of archaeological sites was proposed by Aguilar et al. (2015), by using, as a case-study, the walls of the Chokepukio Archaeological Site near Cusco, Peru.

However, the mechanical methods employed for the seismic analysis of these and other case-studies generally comprise either 


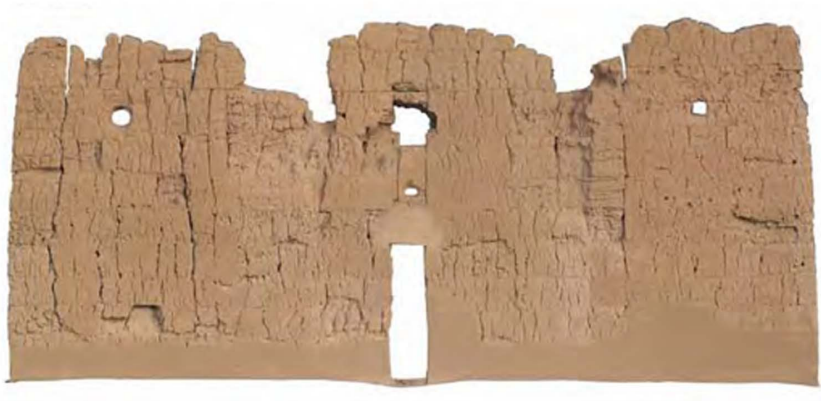

(a)

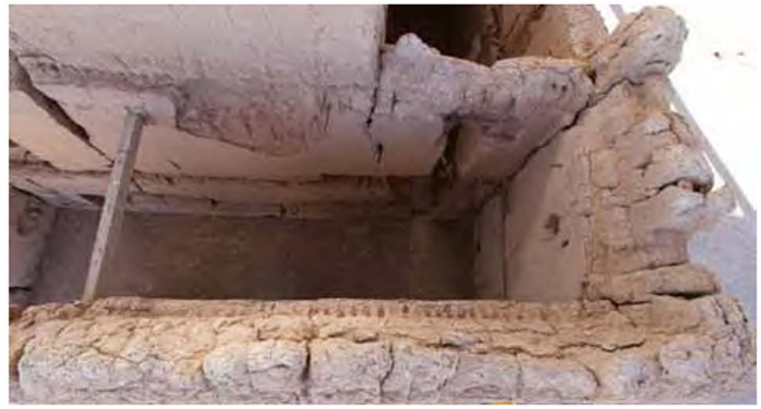

(b)

Fig. 5. (a) West perimeter wall showing large vertical cracks that appear opposite interior partitions and divide façade into several segments. Lens-shaped spalls near base of second wall segment may have occurred in an earthquake (Orthorectified image, Western Mapping Inc.); and (b) North perimeter wall showing extensive axial cracks (parallel to wall surfaces).

simplified analytical models, such as limit analysis and code-based methods (Sassu et al. 2013; Pugi and Galassi 2013), or more complex numerical modeling strategies, such as finite-element analysis and discrete-element methods (Castellazzi et al. 2013; Dimitri et al. 2011; Psycharis et al. 2000; Papatonopoulos et al. 2002). Whereas simplified analytical models tend to incorporate certain dynamic features of the response of the system, they ignore others and, consequently, yield results that tend to be conservative-which can lead to expensive and occasionally unnecessary retrofitting solutions. On the other hand, numerical methods, when calibrated properly, can yield more accurate results but tend to be computationally expensive and time-consuming, especially when the objective of the analysis is to model collapse.

In this paper, a new tool was employed (Mehrotra and DeJong 2018), which can be used to analytically model the collapse of these structures through the use of nonlinear dynamic analysis (rocking dynamics) to directly integrate the equations of motion for the different (local) collapse mechanisms, with implementation in Rhino version 5, making it possible to extend the analysis to complex real-world structural geometries. This yields more refined results than most of the simplified analytical methods, while enabling faster collapse assessment than most numerical methods. Whereas the material characterization of the earthen wall was particularly important for understanding the durability of the materials to environmental factors, such as weathering, for the seismic analysis, the wall was considered to act as a rocking rigid block at failure. As is typical in traditional masonry structures (Heyman 1995), the material stress due to gravity loads is well below the strength of the material. In the largest wall section, a $30.5-\mathrm{cm}$ (1-ft) wide strip of wall supported $5.64 \mathrm{~m}^{3}(60.7 \mathrm{cu} \mathrm{ft})$ of earthen wall. Assuming a density of $2,000 \mathrm{~kg} / \mathrm{m}^{3}(120 \mathrm{lb} / \mathrm{cu} \mathrm{ft})$, and a crushing failure stress of $2.8 \mathrm{MPa}$ (400 psi) for the earth samples at Casa Grande, the width of the hinge at the base of the rocking wall at collapse was estimated to be approximately $38 \mathrm{~mm}$ (1.5 in.) wide. This confirms that the rocking block model is a reasonable approximation for an earthen wall with low stresses.

A seismic assessment of the earthen walls was also conducted by using the procedures outlined in the ASCE 43 standard (ASCE/SEI 2005) and the Italian Building Code [G.U. No. 47 (NTC 2018)], and a qualitative comparison was finally carried out between the results of this assessment and the predictions of the new tool.

\section{Methodology}

Given the presence of large vertical cracks in the great house, as well as substantial section losses at wall bases, the main objective of the seismic vulnerability analysis was to identify the most vulnerable parts of the building to earthquake loading and to provide an initial evaluation of the potential seismic collapse of isolated wall elements, assuming that these wall elements act independently (i.e., are not sufficiently connected to one another). The analysis was focused on evaluating potential collapse, rather than predicting the extent of additional cracking that may occur during an earthquake, for two reasons. First, a main concern of the National Park Service is public safety, and the potential collapse of the structure is of utmost importance. Second, the structure is already severely cracked, and the reliability of detailed computational modeling to accurately predict the extent of further cracking of such a structure during an earthquake is limited.

Vulnerable wall elements were identified as segments isolated from corners or abutting walls (that might brace them) by large cracks or voids, and segments where losses at the wall bases effectively reduced the wall section by $33 \%-50 \%$. In all, eight wall segments were analyzed (Fig. 6); Walls 0a, 2, 3, and 5 were full-height segments isolated by cracks and voids, whereas Wall segments $0 \mathrm{~b}$, 1, 4, and 6 had substantial section losses at their bases. Capturing the actual geometry of these wall segments was essential to accurately model stability against overturning, particularly to capture the effects of out-of-plumb conditions and local loss of material (especially for Wall segments 1, 4, and 6). Models of each wall segment were generated in Rhino by using point cloud data obtained from light detection and ranging (LiDAR) scans of the structure. A mesh was constructed from point cloud data that was used to create a closed solid for each wall segment, capturing the geometry with good precision (Fig. 7).

The overturning stability of each wall segment was assessed by assuming it behaved like a single rocking block with the axis of rotation defined at the base of the wall section (indicated by $O$ in Fig. 8) and that the tensile strength was zero at the rocking interface. It is possible that rocking may not occur at the base of the wall, and horizontal cracks could force rocking at other locations. However, accurate data regarding the depth of the horizontal cracks was not available. With respect to the slenderness of individual wall segments, assuming that rocking occurs at the base of the wall (or where significant degradation has made the wall locally thin) and assuming zero tensile strength to account for potential cracking were conservative assumptions. With respect to scale effects, assuming that rocking will occur at the base of a wall segment may have been slightly unconservative. In other words, rocking that initiated higher in the structure would involve less slender blocks (i.e., more stable), but these blocks would have been of a smaller scale (less stable). As a compromise, wall segments were assumed 

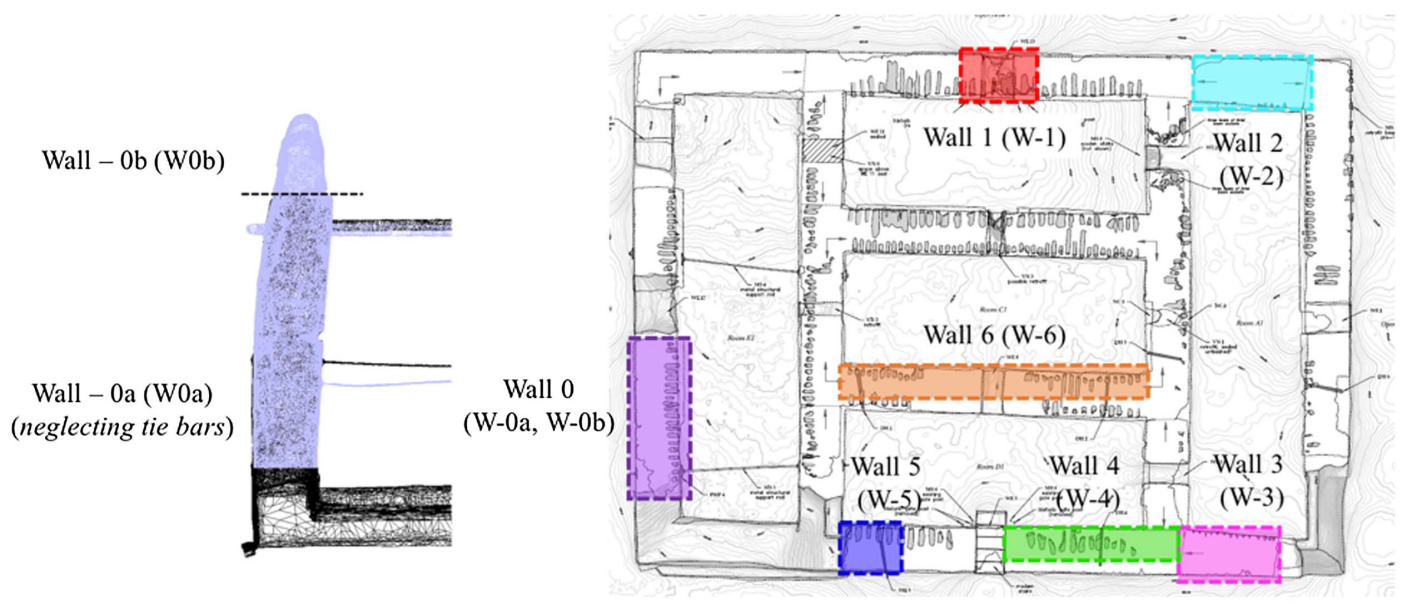

Fig. 6. Location of evaluated wall segments. Elevation provided for Wall 0 to better illustrate two different collapse mechanisms considered for this element.
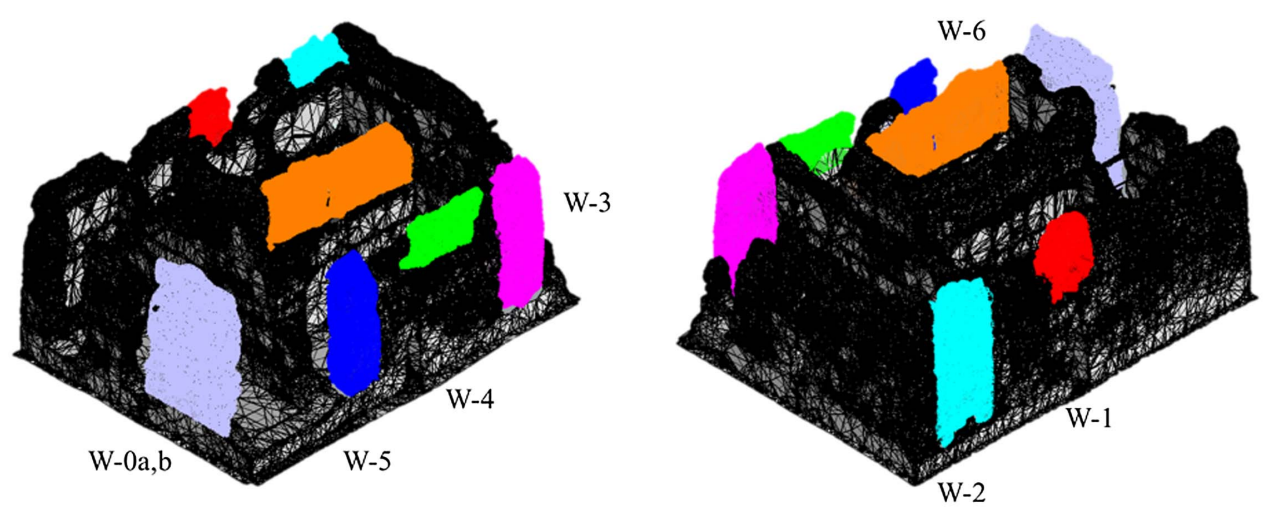

Fig. 7. Three-dimensional computer-aided design models of different wall segments generated by using point cloud data from LiDAR scans of structure.

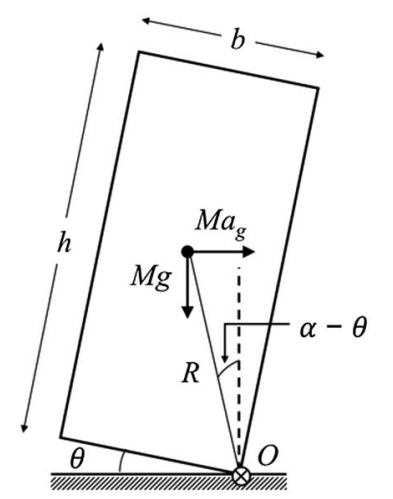

Fig. 8. Geometry of single rocking block.

to rock about their bases but have zero tensile strength. These assumptions were made, in part, to facilitate the demonstration of a new assessment procedure. It is beyond the scope of the study to consider the practically infinite number of potential mechanisms that could occur, though this could be done through automation.

By using these assumptions and the tool developed in Rhino, equations of motion were generated for each wall segment based on the geometry of the wall and the defined axis of rotation (Mehrotra and DeJong 2018). The ability of individual wall segments to resist earthquake loading was evaluated with two different objectives: (1) to determine the relative vulnerability of different parts of the structure (i.e., to identify the most vulnerable parts); and (2) to determine the actual capacity of the wall segments with respect to the expected ground motions on site. This distinction is important because one cannot be certain about the absolute capacity of heritage structures, which contain so many inherent unknowns, but understanding relative capacity is more feasible, assuming that unknowns will cause similar effects for different elements.

\section{Relative Vulnerability to Collapse}

To determine the relative vulnerability of each wall segment, responses to a single sinusoidal ground acceleration pulse of varying frequency $\left(f_{p}\right)$ and amplitude $\left(a_{p}\right)$ were considered, by repeatedly solving the dynamic response of each segment (by using the aforementioned equations of motions) for a range of pulses of different frequency and amplitude. These results were used to generate overturning envelopes (Fig. 9) that predict the rocking response of each wall to a wide range of sinusoidal pulse-type excitations. These plots were broadly divided into safe (i.e., no collapse) and unsafe (i.e., collapse) zones, and indicated, for a pulse with a given frequency and amplitude, which of the wall segments would overturn (with or without impact). 

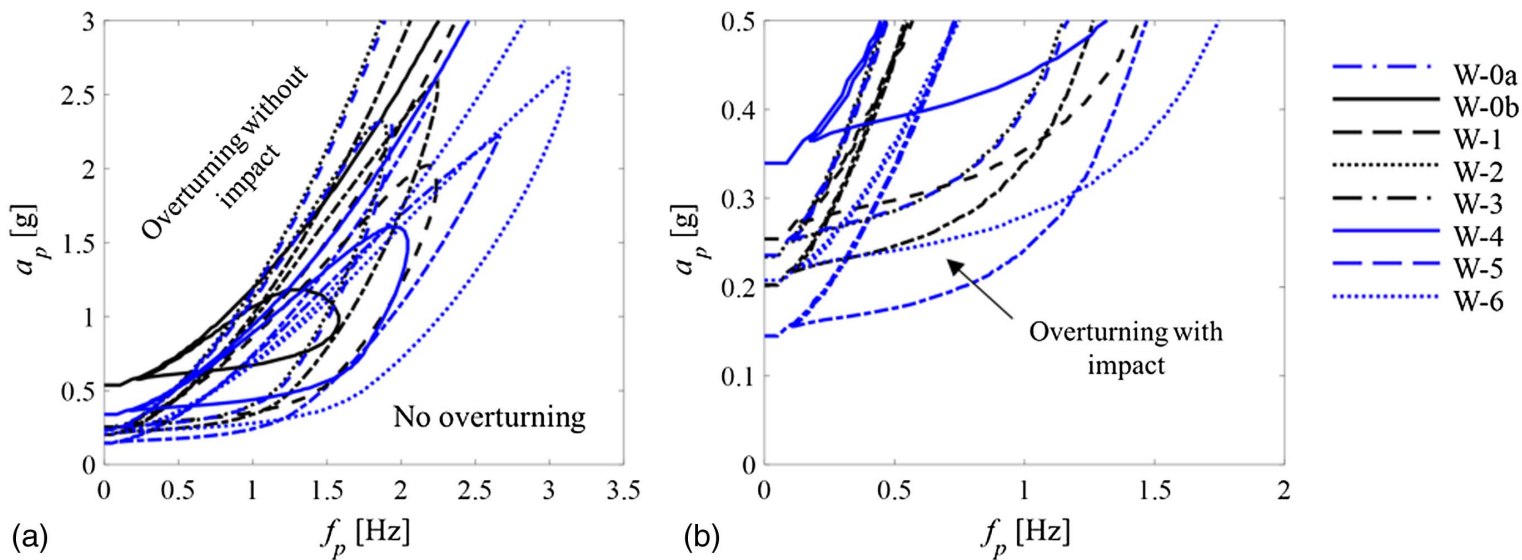

Fig. 9. Overturning envelopes generated for each of different collapse mechanisms. Note that (b) is a zoomed-in version of (a).

Table 2. Seismic design parameters used for definition of response spectrum

\begin{tabular}{ll}
\hline Parameter & Value \\
\hline MCE values & \\
$S_{S}$ & $0.205 \mathrm{~g}$ \\
$S_{1}$ & $0.064 \mathrm{~g}$ \\
$T_{L}$ & $6 \mathrm{~s}$ \\
For site class = D, response spectra defined by & \\
$S_{D S}$ & $0.219 \mathrm{~g}$ \\
$S_{D 1}$ & $0.102 \mathrm{~g}$ \\
\hline
\end{tabular}

Note: $S_{s}$ and $S_{1}=$ maximum considered earthquake spectral response accelerations at short periods and at 1-s period, respectively; $T_{L}=$ long-period transition period; $S_{D S}$ and $S_{D 1}=$ design spectral response accelerations, respectively.

\section{Actual Maximum Response to Expected Ground Motion}

The overturning envelopes provided a good picture of the relative vulnerability of each wall segment, but did not provide a prediction of the maximum response predicted for a specific site with a specific seismic hazard. To achieve this, the rocking response of each wall segment was evaluated by using the procedures found in the ASCE 43 standard (ASCE/SEI 2005) and the Italian Building Code [G.U. No. 47 (NTC 2018)]. These methods predict the maximum rocking response of a structure by using linear-elastic response spectra. To apply these methods, the shape of the design spectrum specified in the International Building Code (IBC) was employed (ICC 2009), with the maximum considered earthquake (MCE) and soil parameters listed in Table 2 . Note that, because a soil investigation was not completed, Site Class D was assumed (ICC 2009). Based on the short- and long-period spectral demands for the MCE, it was inferred that this region had a globally low level of seismic severity, as defined in ASCE 41 (ASCE/SEI 2017).

As in the case of the overturning plots, the tool in Rhino was first used to extract the geometric properties of each of the wall segments, which were then approximated by using equivalent linear elastic oscillators defined by an equivalent period $T$ for both methods. The equivalent period was then used to determine the spectral demand from the linear elastic response spectra for each of the considered wall segments. For a more detailed description of these two methods, see DeJong (2014).

The predictions of both methods were stated in terms of the variation of the peak ground acceleration (PGA) with the maximum rocking angle $\theta$, as illustrated in Figs. 10 and 11 . Note that in both

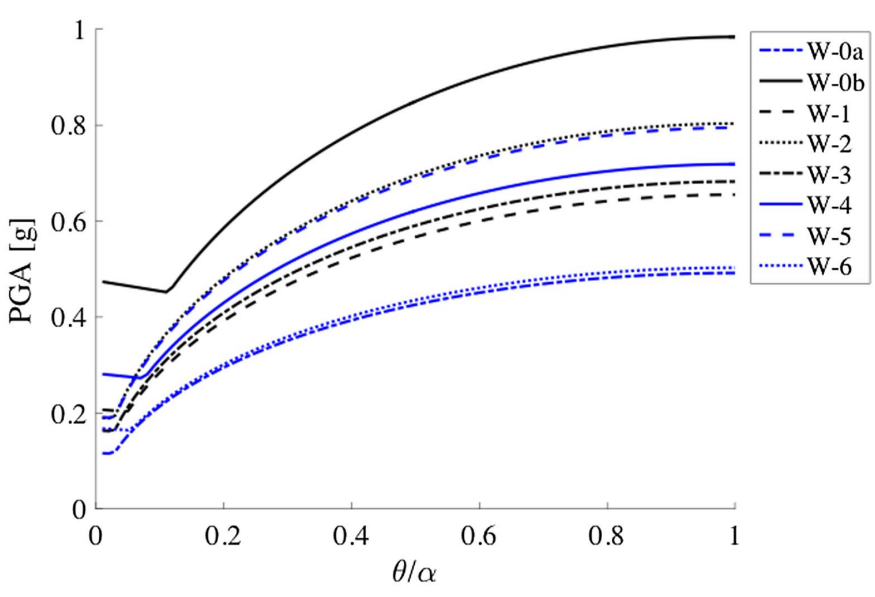

Fig. 10. Variation of maximum rocking rotation $\theta$ with PGA for different wall segments, as derived by ASCE 43 procedure.

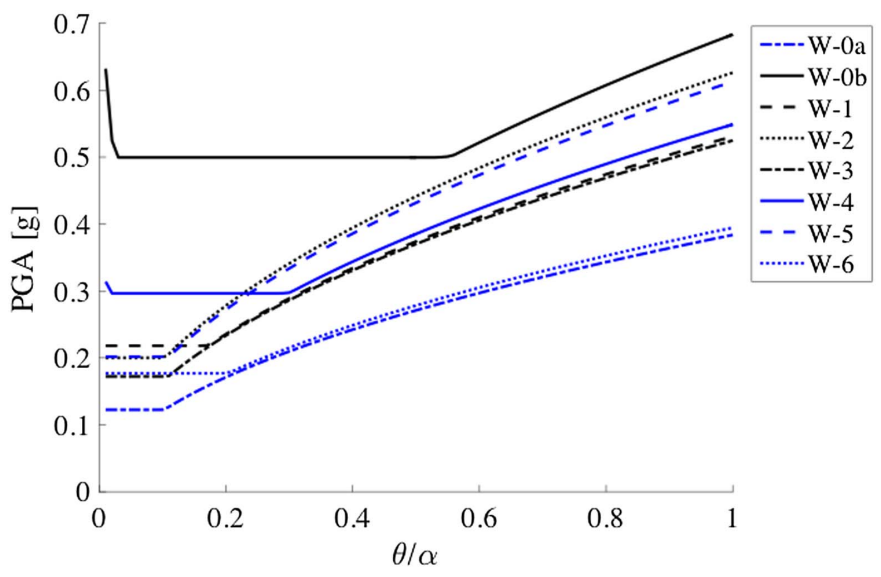

Fig. 11. Variation of maximum rocking rotation $\theta$ with PGA for different wall segments, as derived by the Italian Building Code.

of these plots, $\theta$ is expressed as a fraction of the analytical critical rotation $\theta_{c r}$, i.e., the rotation above which the wall would overturn rather than return to its initial position if released from rest. In the case of the single rocking block mechanism (which is the mechanism assumed for each wall segment), this critical rotation is equal 
to the slenderness angle $\alpha$ of the wall segment under consideration (Fig. 8); that is, $\theta_{c r}=\alpha$, which is also equal to the rotation required for overturning collapse to occur $\theta_{o v}$. However, the two standard/ code methods provide a more conservative definition of this overturning rotation-whereas the Italian code sets this rotation equal to $40 \%$ of the analytical critical rotation $\theta_{c r}$, the ASCE 43 procedure, due to its cryptic definition of the spectral acceleration capacity (and, consequently, the design spectral displacement), sets the overturning rotation equal to approximately $66 \%-75 \%$ of $\theta_{c r}$ [which depends, in turn, upon the aspect ratio $b / h$ of the structure (Fig. 8)] (DeJong 2014). The PGAs required to cause overturning of each wall segment can then be extracted directly from Figs. 10 and 11 . In the case of the Italian code, this is done by determining the PGA for $\theta / \alpha=0.4$ for each of the different walls; whereas, in the case of the ASCE 43 procedure, the ratio of $\theta / \alpha$ corresponding to the overturning displacement first needs to be determined for each wall segment.

According to the IBC, the actual MCE PGA for the site was 0.13 g. As Figs. 10 and 11 indicate, with the exception of Wall segment $0 \mathrm{a}$, no rotation was predicted for this level of ground motion, so the ground motion was increased so that rotation would occur and a safety factor against overturning could be defined.

\section{Results and Discussion}

By using the tool developed in Rhino, the slenderness angle $\alpha$ of each collapse mechanism was first calculated (Table 3). For a rigid structure, $\alpha$-which, for slender structural geometries, is the inverse of the slenderness ratio $h / b$-is equal to the acceleration $a_{g}$ required for rocking to initiate normalized by the acceleration due to gravity $g$ (Fig. 8). Thus, the slenderness angle can be considered a (typically) conservative measure of the minimum ground acceleration required for overturning to occur. Table 3 indicates that wall Segment 0a was the most vulnerable by this measure alone, due to the fact that it was leaning (Fig. 6) more than other wall segments of similar size. The minimum ground acceleration required to cause overturning of this wall is thus $a_{g}=\alpha=0.14 \mathrm{~g}$, which is still greater than the $0.13 \mathrm{~g}$ PGA for the site.

The overturning envelopes for each wall segment in Fig. 9 allow the relative dynamic resilience of each wall to be compared; the lowest curves represent the most vulnerable wall segments. Fig. 9(b) shows that, in the pulse frequency range of $0-1.25 \mathrm{~Hz}$, Wall segment 0a was the most likely to overturn, whereas for frequencies higher than $1.25 \mathrm{~Hz}$, Wall segment 6 was the most vulnerable to collapse. This is because larger walls require a lower frequency pulse to overturn. For higher frequency pulses, the smaller walls are more vulnerable even if they are less slender. After $0 \mathrm{a}$ and 6, Wall segment 3 was the most vulnerable in the lower frequency region, whereas wall Segment 1 was most vulnerable in the higher frequency region. However, this analysis assumed that the interface between the walls and the ground was perfectly rigid, that is-infinitely stiff, with an

Table 3. Computed slenderness $(\alpha)$ for each considered wall segment

\begin{tabular}{lc}
\hline Wall & $\alpha$ \\
\hline $0 \mathrm{a}$ & 0.14 \\
$0 \mathrm{~b}$ & 0.54 \\
1 & 0.25 \\
2 & 0.23 \\
3 & 0.20 \\
4 & 0.34 \\
5 & 0.24 \\
6 & 0.21 \\
\hline
\end{tabular}

infinite compressive strength. In reality, local material deformation (i.e., crushing of the masonry)—which results in an inward shift of the rocking rotation point and consequently a decrease in the maximum rotation the walls can sustain before overturning occurscould make these results unconservative.

Figs. 10 and 11 predict the maximum rocking rotation $\theta$ for a given PGA for each of the different wall segments, as derived by ASCE 43 and Italian code procedures, respectively. In the case of the Italian code, overturning is defined to occur when $\theta / \alpha$ exceeds 0.4 for all considered wall segments; whereas, in the case of the ASCE 43 procedure, the ratio of $\theta_{o v} / \alpha$ corresponding to the overturning displacement first needs to be determined and is listed in Table 4 for each wall segment. The PGAs predicted to cause overturning can then be extracted directly from Figs. 10 and 11 and are listed in Table 5 for both ASCE 43 and Italian code methods. However, given that the PGA of the site is $0.13 \mathrm{~g}$ (IBC), rocking is unlikely to initiate, and overturning is thus unlikely to occur for any of the wall segments considered. Nevertheless, in the case of wall Segment 0a, both methods did predict some rocking of the structure for the PGA of $0.13 \mathrm{~g}$, with the ASCE 43 method predicting a maximum rocking rotation of $0.06 \theta_{o v}$, whereas the Italian code predicted a larger rotation of $0.30 \theta_{o v}$.

A qualitative comparison among the results of the overturning plots (Fig. 9) and those obtained from the two code-based procedures revealed a generally good agreement among the three sets of results in terms of the relative resilience of the different wall segments. All three plots predicted the greatest resistance to collapse (largest PGA required) for Wall segments $0 \mathrm{~b}, 2$, and 5, whereas Wall segments $0 \mathrm{a}$ and 6 were found to be most vulnerable to failure. However, whereas the predictions of the code-based methods were generally more conservative than the overturning envelopes (especially in the higher frequency range), for the lower frequency (longer period) pulses, code-based procedures actually tended to overestimate the dynamic resistance of the walls and were thus unconservative.

Finally, a comparison of predictions of the ASCE 43 and those of the Italian code (Table 5, all wall segments and Fig. 12, using

Table 4. Rotation predicted by ASCE 43 to cause overturning $\left(\theta_{o v}\right)$ normalized by slenderness $(\alpha)$ for each considered wall segment

\begin{tabular}{lc}
\hline Wall & $\theta_{o v} / \alpha$ \\
\hline $0 \mathrm{a}$ & 0.69 \\
$0 \mathrm{~b}$ & 0.86 \\
1 & 0.64 \\
2 & 0.69 \\
3 & 0.71 \\
4 & 0.72 \\
5 & 0.71 \\
6 & 0.68 \\
\hline
\end{tabular}

Table 5. PGA predicted to cause overturning for both ASCE 43 and Italian Building Code methods

\begin{tabular}{lcc}
\hline Wall & ASCE PGA $(\mathrm{g})$ & Italian PGA $(\mathrm{g})$ \\
\hline $0 \mathrm{a}$ & 0.47 & 0.24 \\
$0 \mathrm{~b}$ & 0.97 & 0.50 \\
1 & 0.61 & 0.33 \\
2 & 0.76 & 0.39 \\
3 & 0.65 & 0.33 \\
4 & 0.69 & 0.34 \\
5 & 0.76 & 0.39 \\
6 & 0.48 & 0.25 \\
\hline
\end{tabular}




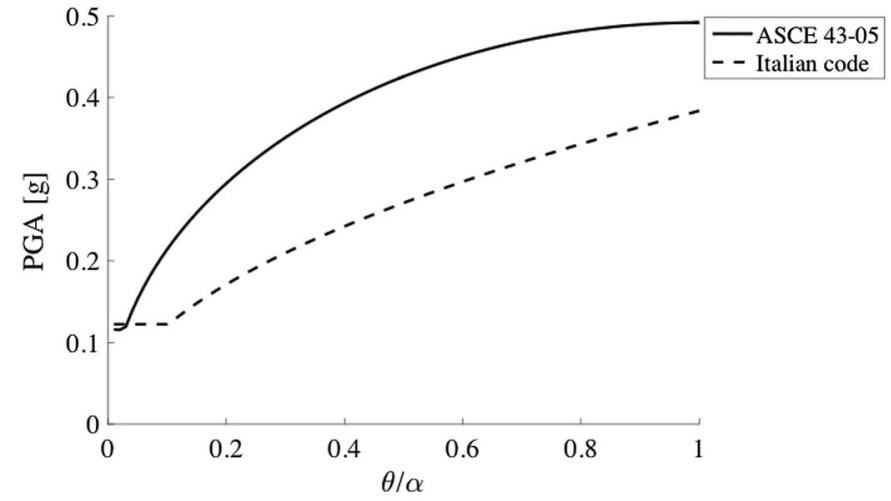

Fig. 12. Comparison of variation of maximum rocking rotation $\theta$ with PGA for Wall segment 0a, as derived by ASCE 43 and the Italian Building Code.

as an example Wall segment 0a) shows that the procedure outlined in the Italian code is generally more conservative than its ASCE 43 counterpart. This is likely because the Italian Code was more directly derived to deal with collapse mechanisms in aging buildings.

The specific findings regarding seismic vulnerability can be summarized as follows:

- Wall segment 0a, which was retrofitted with timber and steel ties in the late-nineteenth century, is the most vulnerable to overturning, primarily due to its inclination. Without this inclination, its stability would be very similar to Wall segment 3 .

- Wall segment 6 is nearly equally vulnerable to overturning as Wall segment 0a.

With the exception of Wall segment 0a, none of the Wall segments were predicted to initiate rocking (i.e., no uplift at all), let alone overturn completely, by using any of the three methods considered (pulse response, ASCE 43, and Italian Building Code).

\section{Conclusions}

This paper summarizes the architectural and seismic analysis of the great house at Casa Grande Ruins National Monument. The architectural analysis included the characterization of the earthen materials used in the construction of walls, including hearting, leveling coats, finishes plasters, and decorative washes. In the hearting, leveling coats, and plasters, secondary calcite was one of the principal matrix cements. These earthen materials have proven to be relatively durable despite several centuries of weather exposure. Since 1906, the great house has been protected by using constructed shelters; the current shelter was designed, in part, by Frederick Law Olmsted, Jr., in 1932, and is itself a National Historic Landmark. Most of the deterioration visible on the great house today predates the construction of the shelters and the period of National Park Service management. Nevertheless, the capacity of the ruin to resist seismic loads has been a longstanding concern.

Regarding the seismic assessment, a new tool that makes it feasible to integrate complicated geometry obtained with LiDAR scan data with dynamic analysis of rocking mechanisms was demonstrated. By using this tool, the capacity of the detailed wall geometries to resist overturning due to earthquake loading was quantified by using both rocking dynamics theory and code-based methods, and the most vulnerable portions of the structure were identified. The results indicate that, by using any of the analytical methods that are currently available, the wall segments are in no danger of collapse as the result of overturning.
However, it must be emphasized that this analysis only considered overturning collapse and not material failure; characterization of the ancient wall materials is ongoing. As one of the next steps in the structural evaluation of the ruin, investigators will use microwave radar and stress wave velocity measurements to produce tomographic images of cracks internal to the walls at three test panel locations; these tests are intended to provide proof of concept for the nondestructive characterization of the internal fracture networks that may adversely affect the structural performance of the walls. Ultimately, the additional materials information (in particular, the material density, elastic modulus, and compressive strength) will be used to refine the seismic assessment results through the incorporation of local material crushing effects, which could further reduce the seismic resilience of the wall segments.

\section{Acknowledgments}

The research presented here was the result of projects conducted through the Colorado Plateau Cooperative Ecosystem Studies Unit between the Department of Anthropology at the University of New Mexico and the National Park Service. Casa Grande Ruins National Monument provided the funding.

\section{References}

Abbott, D. R., C. D. Breternitz, and C. K. Robinson. 2003. "Challenging conventional conceptions." In Centuries of decline during the Hohokam classic period at Pueblo Grande, edited by D. R. Abbott, 3-23. Tucson, AZ: University of Arizona Press.

Aguilar, R., R. Marques, K. Sovero, C. Martel, F. Trujillano, and R. Boroschek. 2015. "Investigations on the structural behavior of archaeological heritage in Peru: From survey to seismic assessment." Eng. Struct. 95 (Jul): 94-111. https://doi.org/10.1016/j.engstruct.2015.03.058.

Andreini, M., A. De Falco, L. Giresini, and M. Sassu. 2013. "Structural analysis and consolidation strategy of the historic Mediceo Aqueduct in Pisa (Italy)." Appl. Mech. Mater. 351-352: 1354-1357. https://doi .org/10.4028/www.scientific.net/AMM.351-352.1354.

ASCE/SEI. 2017. Seismic evaluation and retrofit of existing buildings. ASCE/SEI 41. Reston, VA: ASCE.

ASCE/SEI (Structural Engineering Institute). 2005. Seismic design criteria for structures, systems, and components in nuclear facilities. ASCE/SEI 43. Reston, VA: ASCE.

Bass, A., D. Porter, M. Spilde, M. Guebard, K. Shaum, and N. Ferriola. 2017. "Characterization and comparative analysis of ancient earthen plasters from the American Southwest." MRS Adv. 2 (39-40): 21452178. https://doi.org/10.1557/adv.2017.240.

Casapulla, C., L. Giresini, and P. B. Lourenço. 2017. "Rocking and kinematic approaches for rigid block analysis of masonry walls: State of the art and recent developments." Buildings 7 (69): 1-19. https://doi .org/10.3390/buildings7030069.

Castellazzi, G., C. Gentilini, and L. Nobile. 2013. "Seismic vulnerability assessment of a historical church: Limit analysis and nonlinear finite element analysis." Adv. Civ. Eng. 2013: 1-12. https://doi.org/10 $.1155 / 2013 / 517454$.

DeJong, M. J. 2014. "Rocking of structures during earthquakes: From collapse of masonry to modern design." SECED Newsl. 25 (3): 1-8.

Dimitri, R., L. De Lorenzis, and G. Zavarise. 2011. "Numerical study on the dynamic behavior of masonry columns and arches on buttresses with the discrete element method." Eng. Struct. 33 (12): 3172-3188. https://doi.org/10.1016/j.engstruct.2011.08.018.

Galassi, S., and M. Paradiso. 2014. "BrickWORK software-Aided analysis of masonry structures." IERI Procedia 7: 62-70. https://doi.org/10 .1016/j.ieri.2014.08.011.

Galassi, S., N. Ruggieri, and G. Tempesta. 2018a. "A novel numerical tool for seismic vulnerability analysis of ruins in archaeological sites." Int. J. Archit. Heritage. 1-22. https://doi.org/10.1080/15583058.2018 .1492647 . 
Galassi, S., N. Ruggieri, and G. Tempesta. 2018b. "Ruins and archaeological artifacts: Vulnerabilities analysis for their conservation through the original computer program BrickWORK." In Proc., 11th Int. Conf. on Structural Analysis of Historical Constructions (SAHC 2018), 1839-1848. Braga, Portugal: Univ. of Minho.

Giamundo, V., V. Sarhosis, G. P. Lignola, and E. Cosenza. 2014. "Discrete element modelling of the archaeological colonnade in Pompeii." In Proc., 9th Int. Masonry Conf. 2014. Braga, Portugal: Univ. of Minho.

Guebard, M. C., A. Bass, and D. Porter. 2018. "Colored plasters and cultural meaning at the Montezuma Castle cliff dwelling and Casa Grande Great House.” J. Arizona Archaeol. 5 (2): 101-113.

Heyman, J. 1995. The stone skeleton: Structural engineering of masonry architecture. Cambridge, UK: Cambridge University Press.

ICC (International Code Council). 2009. International building code. Country Club Hills, IL: ICC.

Mehrotra, A., and M. J. DeJong. 2018. "A CAD-interfaced dynamics-based tool for analysis of masonry collapse mechanisms." Eng. Struct. 172: 833-849. https://doi.org/10.1016/j.engstruct.2018.06.053.

Mindeleff, C. 1896. Thirteenth annual report of the Bureau of Ethnology to the Secretary of the Smithsonian Institution, 1891-1892. Edited by J. W. Powell, 179-261. Washington, DC: Government Printing Office.

Minos-Minopoulos, D., D. Dominey-Howes, and K. Pavlopoulos. 2017. "Vulnerability assessment of archaeological sites to earthquake hazard: An indicator based method integrating spatial and temporal aspects." Ann. Geophys. 60 (4): S0445. https://doi.org/10.4401/ag-7157.

NTC (Norme techniche per le costruzioni). 2008. Ministerial Decree dated 14/01/2008. [In Italian.] Rome: Ministero delle Infrastrutture e dei Trasporti.

Papatonopoulos, C., I. N. Psycharis, D. J. Papastamatiou, J. V. Lemos, and H. Mouzakis. 2002. "Numerical prediction of the earthquake response of classical columns using the distinct element method."
Earthquake Eng. Struct. Dyn. 31 (9): 1699-1717. https://doi.org/10 $.1002 /$ eqe. 185 .

Psycharis, I. N., D. J. Papastamatiou, and A. P. Alexandris. 2000. "Parametric investigation of the stability of classical columns under harmonic and earthquake excitations." Earthquake Eng. Struct. Dyn. 29 (8): 1093-1109. https://doi.org/10.1002/1096-9845(200008)29:8< 1093::AID-EQE953>3.0.CO;2-S.

Pugi, F., and S. Galassi. 2013. "Seismic analysis of masonry Voussoir arches according to the Italian Building Code." Int. J. Earthquake Eng. 30 (3): 33-55.

Ruggieri, N., S. Galassi, and G. Tempesta. 2018. “Pompeii's Stabian Baths. Mechanical behaviour assessment of selected masonry structures during the 1st century seismic events." Int. J. Archit. Heritage 12 (5): 859-878. https://doi.org/10.1080/15583058.2017.1422571.

Sassu, M., M. Andreini, C. Casapulla, and A. De Falco. 2013. "Archaeological consolidation of UNESCO masonry structures in Oman: The Sumhuram Citadel of Khor Rori and the Al Balid Fortress." Int. J. Archit. Heritage 7 (4): 339-374. https://doi.org/10.1080/15583058 .2012.665146.

Steen, C. R. 1965. "Excavations in Compound A, Casa Grande National Monument, 1063.” Kiva 31 (2): 59-82. https://doi.org/10.1080 /00231940.1965.11760532.

Van Valkenburgh, S. 1962. "The Casa Grande of Arizona as a Landmark on the Desert, a Government Reservation, and a National Monument." Kiva 27 (3): 1-31.

Vick, E. L. 1973. Investigation and study of materials. Tucson, AZ: Southern Arizona Testing Laboratory.

Wilcox, D. R., and L. Shenk. 1977. The architecture of the Casa Grande and its interpretation. Tucson, AZ: Univ. of Arizona.

Wilcox, D. R., and C. Sternberg. 1981. Additional studies of the architecture of the Casa Grande and its interpretation. Tucson, AZ: Univ. of Arizona Press. 